\title{
Story understanding of a nonexplanatory film affects viewers' premotor activity and empathy for fictional characters
}

This article was published in the following Dove Press journal:

Neuroscience and Neuroeconomics

20 July 2016

Number of times this article has been viewed

\author{
Yukiko Ogawa' \\ Sotaro Shimada ${ }^{2}$ \\ 'Faculty of Social Sciences, Hosei \\ University, Tokyo, ${ }^{2}$ Department of \\ Electronics and Bioinformatics, School \\ of Science and Technology, Meiji \\ University, Kanagawa, Japan
}

Objective: The present study aimed to examine whether the story understanding of a nonexplanatory film affects mirror neuron system (MNS) activity and to discuss the interactive process involved in generating empathy for fictional characters during participants' viewing of the film under natural viewing conditions.

Methods: The material of the experiment was a Japanese film entitled Dolls. It is a nonexplanatory fiction film, in which the two (male and female) main characters show only minimal actions or facial expressions; therefore, the viewers hardly understand the story until it has been developed to some extent. We measured twelve participants' MNS activity by using near-infrared spectroscopy during the viewing of the first 20 minutes of the film. Additionally, we measured the brain activity while performing their own hand and leg motions after viewing the film to identify their motor cortices.

Results: The results showed that the viewer's MNS activity increased gradually as the introductory part of the story developed. Subsequent analyses revealed a significant increase in MNS activity in the later chapters of the film at the right premotor and supplementary motor cortices $(P<0.05)$. Furthermore, there was a significant correlation between the MNS activity and the Interpersonal Reactivity Index scores $(P<0.05)$.

Conclusion: These results indicate that the viewer's MNS activity was enhanced by the story understanding of a fiction film. We suggest that MNS activity during viewing fiction films can be used as a measure of how much the story, and rhetoric of a narrative, induces empathy in the viewers even if characters show only minimal actions or facial expressions.

Keywords: mirror neuron system, MNS, empathy, nonexplanatory film, story understanding, premotor cortex

\section{Introduction}

Empathy has been defined as the state where perceivers represent the same emotion they are observing or imagining in another person with full awareness that the source of their own experience is the other's emotion. ${ }^{1}$ All factors that constitute, or stem from, the concept of empathy have been explored under various conditions in neuroscience. Such enthusiastic exploration of the mechanisms of empathy reflects the perception that empathy is generally understood to be the basis of humans' prosocial nature and the cognitive capacity needed for altruistic behavior. ${ }^{2-4}$ Taking into account the requirement for investigation of empathy based on social interests and real-life issues, the present study focused on relationships between empathy and depictions in a fiction film, an issue which has rarely been investigated. By measuring brain activity of participants viewing a fiction film under natural viewing conditions with no
Correspondence: Yukiko Ogawa Faculty of Social Sciences, Hosei University, 4342 Aihara-Machi, Machida-shi, Tokyo 194-0298, Japan Email yukiko_ogawa@jazz.odn.ne.jp 
experimental interventions, the present study examines and discusses the interactive process involved in generation of empathy for the film.

Using a methodologically inventive approach shared by Hasson et $\mathrm{al}^{5}$ and Bartels and Zeki, ${ }^{6}$ natural viewing conditions were adopted for this experiment in order to avoid the potential problem of using data obtained from an experiment performed under limiting conditions in order to discuss cognitive states present during natural viewing. Thus, the present study was conducted based on the hypothesis that the mirror neuron system (MNS), which is activated by a network of neurons in multiple brain areas involved in the motor function of the subject when the subject performs an action and observes the same action being performed by the target, is the main neural basis for emotional empathy. ${ }^{7-9}$ Overlap of cortical activation during self-produced actions and actions observed from others can be interpreted as "isomorphic" affective states; that is, sharing of the same emotion by the perceiver and others. ${ }^{10-14}$ Research in MNS suggest that actions and facial expressions are generally considered to be the main information to be provided by others to evoke such isomorphic affective states in the perceiver. ${ }^{15,16}$ However, based on a presumption that the MNS activity can be induced during the viewing of a fiction film by information obtained not only from relevant actions and facial expressions of characters in the film but also from elements that do not belong to the characters, such as story and directorial technique, the present study intentionally used, as the material of the experiment, a nonexplanatory fiction film in which characters show only minimal actions or facial expressions while providing intentionally limited lingual information that might complement their constrained actions or facial expressions.

"Minimization of explanatory elements" is a common world-class filmmaking technique, which is supported by internationally respected film directors. How do mirroring related to the MNS and neural activation that infers the mental state of others (mentalizing) interact to construct cognition in the brain of the perceiver viewing a film with minimal explanatory elements? To investigate this point, we employed Dolls, directed by Takeshi Kitano (http://www.office-kitano. co.jp/dolls/), which was judged to be the most suitable material for the present study because of its tragic love story which would likely induce empathy for the characters while explanatory elements were used as little as possible.

In associated fields such as psychology and cognitive science, only a few studies of empathy in fiction films have been conducted. ${ }^{17-19}$ Meanwhile, in the field of neuroscience, a small core of researchers has been researching this issue intensively, building a solid foundation for further research. ${ }^{5,20-22}$ Most natural viewing studies emphasize the necessity of doing experiments without strict constraints, in which participants are allowed to focus naturally on the presented stimulus, while most of those utilizes functional magnetic resonance imaging. In the present study, we use near-infrared spectroscopy (NIRS), which is a noninvasive neuroimaging method that measures changes in the oxyhemoglobin (oxy-Hb), deoxyhemoglobin (deoxy-Hb), and total-hemoglobin (total-Hb) concentrations. ${ }^{23}$ NIRS has been applied to various cognitive studies, including sensorimotor, visual perception, language, and executive functions; developmental studies; and clinical studies. ${ }^{24-28}$ NIRS makes it possible to measure brain activity in a nearly "natural state" with almost no physical constraints given to participants. This is the methodological superiority that is not fulfilled by the experiment that utilized functional magnetic resonance imaging.

Our greatest concern here is not to generally specify the activated brain regions during film viewing but to show the process of empathy by the data extracting overlap of fictional characters and perceiver's motor cortex. The standpoint of the present study is based on the knowledge that MNS activity contributes to represent a person's action that in turn leads to a deeper understanding of the internal states such as intentions or emotions of another person. ${ }^{29}$ Moreover, the present study explores how neuroscience can be used to investigate films further in an artistically valid manner. The methodology of neuroscience is expected to provide useful suggestions as to how films are studied.

\section{Methods}

\section{Participants}

Twelve healthy adult individuals (six females and six males, aged 19-23 years; mean \pm standard deviation: $21.08 \pm 1.44$ years) participated in the experiment. None of these participants had watched this experimental material before, and all but one did not know the film, which was confirmed on the basis of the verbal report. All participants were undergraduate or graduate students at School of Science and Technology, Meiji University, and had normal vision and hearing. They were paid a small fee for participating in this experiment. Written informed consent was obtained from all participants. The experiment was approved by the ethics committee of the School of Science and Technology, Meiji University, and was conducted in accordance with the principles and guidelines of the Declaration of Helsinki. 


\section{Experimental environment}

A television monitor 47LW5700-JA (47" display size; screen inside diameter: $58.6 \mathrm{~cm}$ height $\times 104.3 \mathrm{~cm}$ width) made by LG was used for the experiment. Participants' seating consisted of swivel chairs with arms, placed so that the central axis of their legs was above a line $140 \mathrm{~cm}$ away from the front of the TV screen. This allowed a distance of $\sim 140 \mathrm{~cm}$ between the screen and the eyes of each participant. The experiment was performed in a laboratory with the lights dimmed.

\section{Material}

The material of the experiment was a Japanese film entitled Dolls (directed by Takeshi Kitano, 2002) depicting Matsumoto (Hidetoshi Nishijima), who rejects his engagement with his fiancée, Sawako (Miho Kanno). In despair, she loses her sanity and ends up in a doll-like state, losing her mind and ability to speak. Seeing her suffering, Matsumoto decides to share her destiny for the rest of his life. The film shows his quiet masculinity and his and Sawako's strong love for each other together with a sense of emptiness. Since the film's focus is on these two main characters "wandering together silently", it appears to provide little information needed to evoke empathy among viewers. However, this extremely limited information is conversely likely to stir the imagination of viewers.

The individual chapters of the film are summarized as follows: Chapter 1 (0:00:00-0:00:44) shows the title and opening credits. Chapter 2 (0:00:45-0:06:34) consists of scenes from "The Courier of Hell” (Meido No Hikyaku), a Bunraku (Japanese puppet drama) play. Chapter 3 (0:06:35-0:08:47) adopts the narrative technique of antichronological order, showing the story's present. Sawako, who has lost her mind and ability to speak, and Matsumoto, who has decided to share her destiny, are wandering under flowering cherry trees, bound together by a piece of red string. In Chapters 4 and 5 , which are back in time, the background of the story is narrated. The main setting of Chapter $4(0: 08: 48-0: 12: 01)$ is a church where Matsumoto, who has broken up with Sawako, is about to marry the daughter of his company's president. In Chapter 5 (0:12:02-0:16:21), Matsumoto leaves the church after being informed of Sawako's suicide attempt and drives to the hospital to which she has been admitted. Chapter 6 (0:16:22-0:21:00) shows a hospital scene where Matsumoto sees Sawako, who appears dissociated.

Perceivers are generally expected to respond to the visual images and story summarized above as follows: In Chapter 3, where the two main characters appear for the first time, perceivers are most likely to be unable to understand the meaning or background of their wandering because of the inverted structure of the chapter. When viewing Chapter 4, which shows friends talking while visiting Matsumoto to attend his wedding ceremony, and scenes from the story's past, associated with their conversations, perceivers may gradually understand what has happened to the couple. In Chapter 5, where past images of their golden time together, and shots of Sawako losing her sanity are shown with a prolonged shot of Matsumoto displaying a serious facial expression while driving to the hospital, perceivers with growing understanding of the couple's situation may begin to respond emotionally to it. In Chapter 6, where the couple finally see each other, they both remain speechless and motionless without even making eye contact. There is a prolonged, silent shot of Sawako, who may or may not recognize him, and Matsumoto, who fixes his eyes on her in a peculiar way. In the course of narrative development from Chapter 5 to this scene in Chapter 6, perceivers are expected to have mixed feelings with emotional empathy ${ }^{30}$ for Sawako while blaming Matsumoto who has unreasonably betrayed her trust in him and cognitive empathy ${ }^{30}$ evoked while trying to discern the inner feelings of Matsumoto.

\section{NIRS measurement}

A multichannel NIRS unit operating at 780-, 805-, and 830nm wavelengths (OMM-3000, Shimadzu, Kyoto, Japan) was used to measure temporal changes in concentrations of oxy$\mathrm{Hb}$, deoxy-Hb, and total-Hb. Sixteen optodes were placed on the left and right motor regions in a lattice pattern to form 24 channels for each hemisphere, centered at the $\mathrm{C} 3$ and $\mathrm{C} 4$ of the EEG 10-20 system ( $9 \times 9 \mathrm{~cm}$ square area). Each channel consisted of one incident optode and one detecting optode located $3 \mathrm{~cm}$ apart from the incident optode. The sampling rate was $\sim 10 \mathrm{~Hz}$. An extensive description of NIRS theory can be found elsewhere. ${ }^{23}$

\section{Procedure}

Participants were seated and then viewed the opening of Dolls (0:00:00-0:21:00; Chapter 1 to the middle of Chapter 6) on the screen in a natural setting while their brain activity was measured using NIRS. The experiment was done individually. The NIRS data obtained during Chapter 3 (0:06:35 ) and subsequent chapters, in which the two main characters appeared, were analyzed. After viewing the film, participants were instructed to clasp and unclasp their handset about 1-2 $\mathrm{Hz}$ (hand motion condition for 5 seconds) or tap their feet while sitting on the chair (leg motion condition for 5 seconds) 
so that we could identify activated regions of interest (hand and leg motor region in the motor cortex) in the present study. Their brain activity during these motions was also measured. According to the experiment protocol, a 20-second rest was allowed to settle their brain activity before starting each motion, and a set consisting of a 5-second pretask rest, 5-second (hand or leg) motor task, and 10-second post-task rest was repeated six times. A 60 -second rest was provided between a hand motion session and a leg motion session.

\section{Data analyses}

The film was analyzed each second and flagged according to whether it included any of the following ten regressors to create a design matrix: 1) main male character, 2) main female character, 3) two main characters, 4) other characters, 5) dialogue, 6) switch of shots, 7) switch to the past or the future and omission of time in the present tense, 8) motion of camera, 9) motion of objects, and 10) slow motion. The regressors of interests were 1, 2, and 3, and the regressors of no interests were 4-10. Note that we flagged regressor 3 (two main characters) only when both male and female main characters appeared on the screen at the same time, and unflagged regressors 1 and 2. NIRS data obtained during film viewing were analyzed for each participant using a general linear model, and a group test was performed for each chapter in terms of the three regressors of interest to examine whether there was any significant activation for each channel. Similarly, NIRS data obtained during the hand and leg motion sessions were also analyzed for each channel.

Participants were asked to complete the Interpersonal Reactivity Index (IRI) as well as the questionnaire survey with regard to Dolls after NIRS data acquisition. In Davis' IRI, conventional emotional empathy scale that cognitive components were downplayed was reexamined and reconstituted into four dimensions by both cognitive and emotional components. ${ }^{31,32}$ Perspective-taking measures the tendency to spontaneously adopt the psychological point of view of others. Fantasy measures respondents' tendencies to transport themselves imaginatively into the feelings and actions of fictional characters. Empathic concern assesses feelings of compassion and concern for unfortunate others. Personal distress assesses "self-oriented" feelings of personal anxiety and unease in response to others' tense experiences. Perspective-taking and fantasy were designed to measure the cognitive components of empathy. Empathic concern and personal distress were designed to assess the emotional components of empathy. Higher scores of each dimension represent higher empathic tendencies. IRI is frequently used as a measure for social cognitive abilities or empathy in functional neuroimaging literature. ${ }^{33-35}$

\section{Results}

From the analysis of data obtained during the hand and leg motion sessions, five channels of left hemisphere (ch-1, 2, 5, 13, 15) and three channels of right hemisphere (ch-26, 30, 34) were identified as motor cortices $(P<0.05$, Bonferroni-corrected;

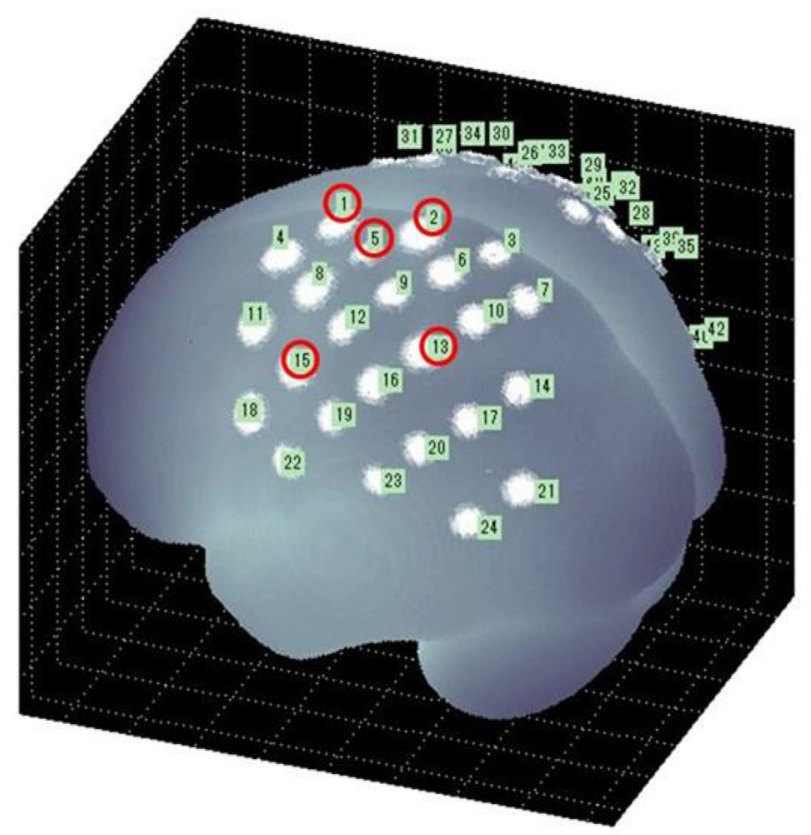

Figure I The channels of the left hemisphere functionally identified as motor areas (red circles).

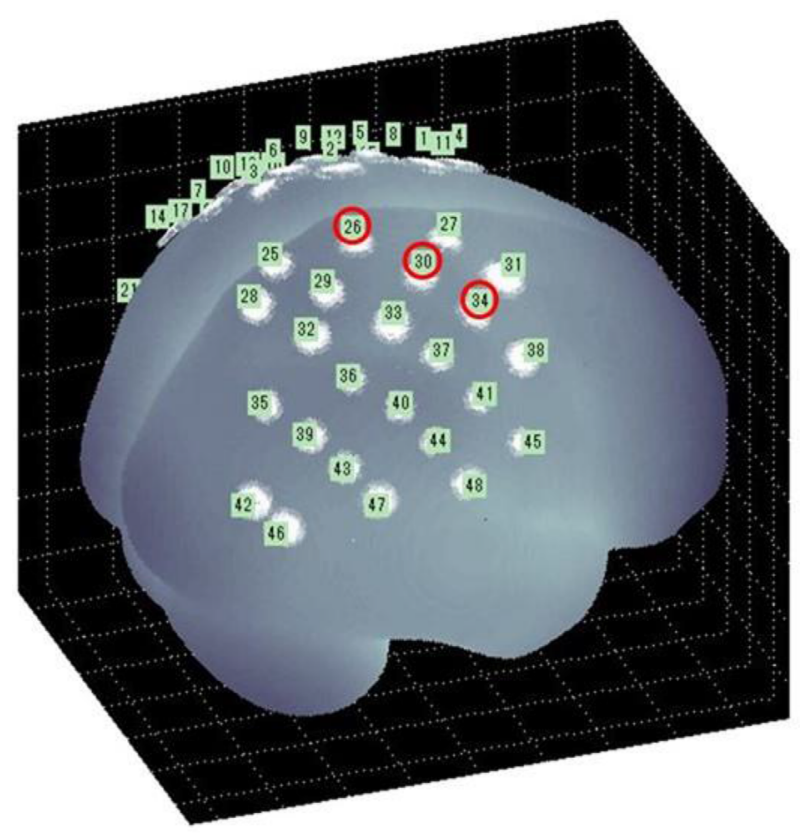

Figure 2 The channels of the right hemisphere functionally identified as motor areas (red circles). 
Figures 1 and 2). For these eight channels, a one-factor analysis of variance was performed for changes in motor cortex activity in chapters in terms of the three regressors related to the main characters. Findings included the main effect of the chapter, in the right premotor cortex and supplementary motor cortex for the regressors: the main female character (ch-34: $F[3$, 33] $=6.169, P<0.05$, false discovery rate-corrected) and the two main characters (ch-30: $F[3,33]=5.016, P<0.05$; ch-34: $F[3$, 33] $=7.054, P<0.01$, false discovery rate-corrected; Figures 3 and 4). Using a Tukey's honestly significant difference test to compare activity among chapters, a significant difference was detected in the amplitude of activity between Chapters 5 and 6 in the two main characters' condition at ch-30 and ch-34 (Chapter $5<$ Chapter 6: ch-30; $P<0.05$, ch-34; $P<0.01$ ). There was a significant correlation between the brain activity for the two main characters' condition in Chapter 6 at ch-30 and the fantasy in $\operatorname{IRI}^{31,32}(r=0.589, P<0.05$; Figure 5).

In a questionnaire survey conducted after the experiment, the following participants' comments were obtained: "I had no idea what the two main characters are doing and what this depiction represents" (Chapter 3), "I gradually began to understand what is going on in this story" (Chapters 4 and 5), and "I finally realized that this scene would be the beginning of this story, and the story so far made sense to me" (Chapter 6). These comments showed that the participants' story understanding was advanced gradually; that is, they came to understand the story plot and structure when their viewing arrived at Chapter 6 while it was vague at the beginning of the film (Chapter 3).

\section{Discussion}

Takeshi Kitano, the director of Dolls, commented that he basically wanted to show the beauty of the scenery in the film. ${ }^{36}$ In addition to limited dialogues and explanation of situations, the film highlights the blank expressions of the two main characters, as if refusing to provide viewers with any information about their current emotions and thus emphasizes a gap between their happy days in the past and their current wrecked state. Such techniques contribute to creating a unique atmosphere in the film. Chapter 2 shows Bunraku puppets as the connotation of the story in the film, and Chapter 3 shows a symbolic image of the story for the first time; that is, the couple wandering, bound by a red string, through four seasons after experiencing core events of the story that have caused this wandering. The flow of time of associated events leading to the behavior or situation is depicted little by little in Chapter 4 and the subsequent chapters. Seeing the opening part of the film, which uses a narrative structure called analepsis, perceivers are obliged to experience a cognitive process where they do not clearly understand the behavior or situation

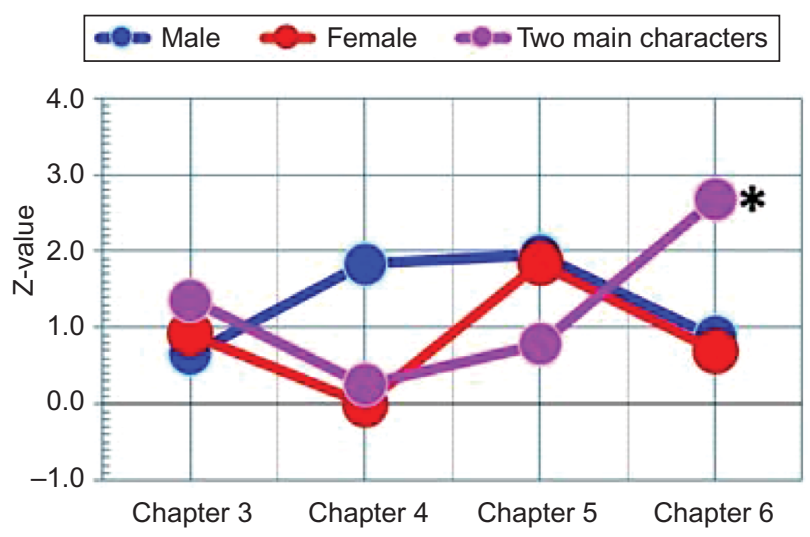

Figure 3 Activation changing of ch-30 (right premotor cortex and supplementary motor cortex).

Notes: Figure indicates results of one-factor analysis of variance and significance level. $* P<0.05$. Male, main male character; Female, main female character.

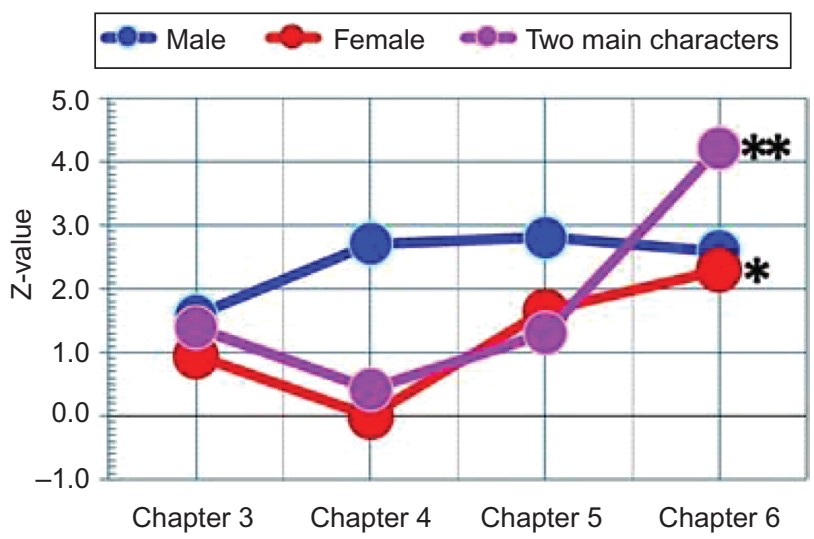

Figure 4 Activation changing of ch-34 (right premotor cortex and supplementary motor cortex).

Notes: Figure indicates results of one-factor analysis of variance and significance level. $* P<0.05$ and $* * P<0.0$ I. Male, main male character; Female, main female character.

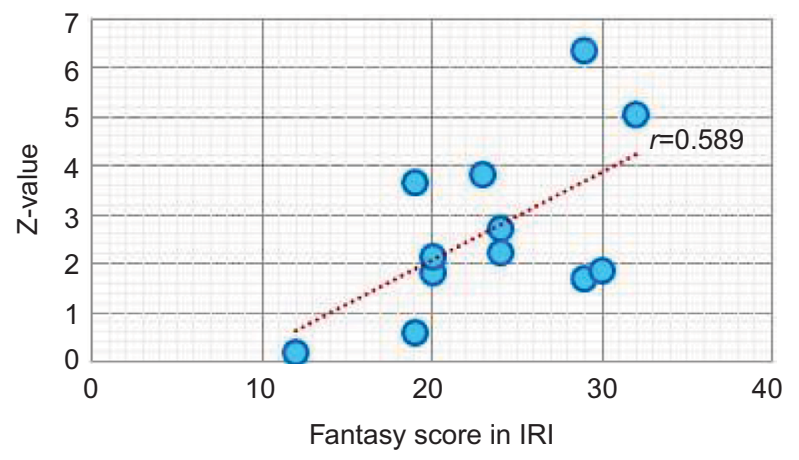

Figure 5 Correlation between the brain activity for two main characters' condition in Chapter 6 at ch-30 and the "Fantasy" in Interpersonal Reactivity Index.

Abbreviation: IRI, interpersonal reactivity index.

of the two main characters in Chapter 3. The intentionally limited dialogues and facial expressions of the couple also increase stress on perceivers as they struggle to understand the narrative situation. Due to the temporal distortion of the 
story and small amount of information provided, perceivers are required to speculate about the emotions and inner states of the couple while viewing the scene.

Responding to this material in the experiment, activations of the right primary motor cortex (ch-26) and right premotor cortex/supplementary motor cortex (ch-30, ch-34) were significantly enhanced. This result indicates that mirroring occurred in the brain of participants trying to understand the emotions and inner states of the couple by simulating the narrative situation using their own motor program. To a question, "What were you thinking while viewing each chapter?" in a questionnaire survey conducted after the experiment, eight of the 12 participants replied, "I was wondering what Matsumoto was thinking", and six participants replied, "I felt sorry for Sawako". These responses demonstrate that participants were obliged to undergo cognitive processing to speculate about the emotions and inner states of the couple throughout the time of viewing the film and that, as a result, their sympathy for Sawako, that is, emotional empathy, was evoked.

However, taking into account the observation that motor cortical activation in the two main characters' condition remained low during Chapters 3-5, in contrast to the markedly enhanced activation in Chapter 6 , and that a significant difference in activation was detected between Chapter 6 and Chapters 3-5 using multiple comparisons, the brain activity system that serves as the basis for the above cognitive processing was likely to have greatly altered at the beginning of Chapter 6. While viewing Chapters 3-5, participants had to construct their basic understanding of Matsumoto and Sawako by speculating to some degree about the emotions and inner states of the couple, consolidating the limited information provided, and simulating the flow of the narrative. This processing, termed cognitive empathy, is handled by a highly sophisticated cognitive perspective-taking system related mainly to the medial prefrontal cortex, and this distinct inner process, mentalizing, is believed to interact with mirroring. ${ }^{37,38}$ When participants viewed the film from Chapters 3 to 5 , although premotor cortical activation remained comparatively low, the brain activity in regions involved in mentalizing was thought to be activated as the basis for cognitive empathy requiring conscious efforts. During Chapter 6, where Matsumoto saw Sawako in the presentation of the film's diegesis, mentalizing became less necessary and was anticipated to switch to MNS activity instead, which can be activated less consciously and more automatically compared with mentalizing, leading to the generation of emotional empathy. In this way, it can be said that the necessity to select a fiction film with the temporal distortion of the story such as Dolls was high for the present study. This is because it was necessary to pose a riddle to the viewer at the beginning of the story in order to produce a shift in brain activity toward emotional empathy after introspective thoughtful reasoning had been stimulated in the viewer.

Given that activation of the MNS generally requires the perceiver's observation of the actions and facial expressions presented by the target, the present study's presumption is supported by the experimental results-participants displayed activation of the MNS when viewing Chapter 6, which showed the couple seeing one another, despite the fact that little emotion or information about the inner state of the couple was presented by the two main characters, even in this chapter. This result may have been attributable to effects of the story (presentation of context) and of the nonexplanatory style of the film. While gradually developing the story, the film did not provide perceivers with all the information needed for their understanding of the story and substantially allowed them to use their own imagination to make up for the lack of information. This skillful approach to the film's structure created by combining the story and presentation has likely contributed to the enhancement of the "fantasy" as a significant activity. From a neuroscientific perspective, world-class filmmaking may aim to activate conscious, reflective, and inferential thinking and then early automatic and unconscious process of understanding emotion in a successive or complex manner by skillfully controlling how information or how much information is provided to perceivers, as shown in the film Dolls.

The present study challenges an issue with few examples in the field of neuroscience, to investigate activation change during film viewing and the suggested coordinated relationship between story understanding, MNS, and empathy. This is the meaningful suggestion at the point where the perceiver's activation change of MNS under the condition that another person targeted for empathy lives in the fictional world was measured. The results of the present study will greatly contribute to the progress of neuroscience in the coming years, which mainly takes interest in the characteristics and activation conditions of the MNS. However, the present study employed a small sample, and as several relevant measurement variables could not be managed, there is a limitation of the generalizability of the result, for example, no control of physiologic variables, such as hormone level and menstruation in females. In future studies that succeed the purpose, methodology, and results of the present study, those flaws should be overcome progressively, and the 
generalizability of the result must be raised definitely. The material like a film, with a great deal of variables, is difficult to fit into the paradigm of experimental science. This kind of natural viewing study in psychology or cognitive science that is going to clarify cognition when viewers watch any film itself as the finished product, not image edited or processed for experiments, has two weak points as the experimental science, namely, 1) variables of film side cannot be controlled strictly and 2) the data cannot be acquired without depending on a subjective report of viewers. Correspondingly, there is no doubt that the methodology of neuroscience, which provides credible evidence through physical measurements of brain activity, overcomes such limitations of previous film cognition research in psychology or cognitive science and will make it possible to develop detailed discussions.

\section{Conclusion}

In order to examine and discuss the interactive process of empathy related to a fiction film, the brain activity of participants was measured using NIRS as they viewed Dolls, a nonexplanatory Japanese film. Experimental results showed that the premotor cortical activation remained relatively low when participants viewed the film from Chapters 3 to 5 , which required them to speculate and introspectively understand the behaviors and situations of the two main characters, from their past to the narrative present, but in Chapter 6, where the couple see one another in the narrative present, the premotor cortical activation was markedly and significantly increased. The present study suggests that the brain activity system that serves as the basis for empathy for fictional characters switched substantially from the cognitive perspective-taking system to the MNS, in other words, from mentalizing to mirroring.

\section{Acknowledgment}

This research was partially supported by Grant-in-Aid for Scientific Research (KAKENHI; 25700015) from Japan Society for the Promotion of Science (JSPS).

\section{Disclosure}

The authors report no conflicts of interest in this research.

\section{References}

1. de Vignemont F, Singer T. The empathic brain: how, when and why? Trends Cogn Sci. 2006;10(10):435-441.

2. Moll J, Zahn R, de Oliveira-Souza R, et al. Impairment of prosocial sentiments is associated with frontopolar and septal damage in frontotemporal dementia. Neuroimage. 2011;54(2):1735-1742.

3. Inagaki TK, Eisenberger NI. Neural correlates of giving support to a loved one. Psychosom Med. 2012;74(1):3-7.
4. Morelli SA, Rameson LT, Lieberman MD. The neural components of empathy: predicting daily prosocial behavior. Soc Cogn Affect Neurosci. 2014;9(1):39-47.

5. Hasson U, Nir Y, Levy I, Fuhrmann G, Malach R. Intersubject synchronization of cortical activity during natural vision. Science. 2004; 303(5664):1634-1640.

6. Bartels A, Zeki S. Brain dynamics during natural viewing conditions - a new guide for mapping connectivity in vivo. Neuroimage. 2005;24(2):339-349.

7. Rizzolatti G, Fogassi L, Gallese V. Neurophysiological mechanisms underlying the understanding and imitation of action. Nat Rev Neurosci. 2001;2(9):661-670.

8. Avenanti A, Bueti D, Galati G, Aglioti SM. Transcranial magnetic stimulation highlights the sensorimotor side of empathy for pain. Nat Neurosci. 2005;8(7):955-960.

9. Iacoboni M. Imitation, empathy, and mirror neurons. Annu Rev Psychol. 2009;60:653-670.

10. Wicker B, Keysers C, Plailly J, Royet JP, Gallese V, Rizzolatti G. Both of us disgusted in My insula: the common neural basis of seeing and feeling disgust. Neuron. 2003;40(3):655-664.

11. Gallese V, Keysers C, Rizzolatti G. A unifying view of the basis of social cognition. Trends Cogn Sci. 2004;8(9):396-403.

12. Singer T, Seymour B, O'Doherty J, Kaube H, Dolan RJ, Frith CD. Empathy for pain involves the affective but not sensory components of pain. Science. 2004;303(5661):1157-1162.

13. Jackson PL, Brunet E, Meltzoff AN, Decety J. Empathy examined through the neural mechanisms involved in imagining how I feel versus how you feel pain. Neuropsychologia. 2006;44(5):752-761.

14. Shimada S, Oki K. Modulation of motor area activity during observation of unnatural body movements. Brain Cogn. 2012;80(1):1-6.

15. Goldman AI, Sripada CS. Simulationist models of face-based emotion recognition. Cognition. 2005;94(3):193-213.

16. van der Gaag C, Minderaa RB, Keysers C. Facial expressions: what the mirror neuron system can and cannot tell us. Soc Neurosci. 2007;2(3-4):179-222.

17. Oliver MB, Weaver JB, Sargent SL. An examination of factors related to sex differences in enjoyment of sad films. J Broadcast Electron Med. 2000;44(2):282-300.

18. Bartsch A, Appel M, Storch D. Predicting emotions and meta-emotions at the movies: the role of the need for affect in audiences' experience of horror and drama. Commun Res. 2010;37(2):167-190.

19. Ogawa Y, Kanai A. Rhetoric of biopic and viewer's reconsideration: a verification that how TARO's enjoyment and YUMEJI's irrational cutting cause an effect in concept reconsideration. Cogn. Stud. 2013;20(2):224-248.

20. Hasson U, Furman O, Clark D, Dudai Y, Davachi L. Enhanced intersubject correlations during movie viewing correlate with successful episodic encoding. Neuron. 2008;57(3):452-462.

21. Lahnakoski JM, Salmi J, Jääskeläinen IP, et al. Stimulus-related independent component and voxel-wise analysis of human brain activity during free viewing of a feature film. PLoS One. 2012;7(4):e35215.

22. Pamilo S, Malinen S, HlushchukY, Seppa M, Tikka P, Hari R. Functional subdivision of group-ICA results of fMRI data collected during cinema viewing. PLoS One. 2012;7(7):e42000.

23. Scholkmann F, Kleiser S, Metz AJ, et al. A review on continuous wave functional near-infrared spectroscopy and imaging instrumentation and methodology. Neuroimage. 2014;85(1):6-27.

24. Leff DR, Orihuela-Espina F, Elwell CE, et al. Assessment of the cerebral cortex during motor task behaviours in adults: a systematic review of functional near infrared spectroscopy (fNIRS) studies. Neuroimage. 2011;54(4):2922-2936.

25. Ferrari M, Quaresima V. A brief review on the history of human functional near-infrared spectroscopy (fNIRS) development and fields of application. Neuroimage. 2012;63(2):921-935.

26. Dieler AC, Tupak SV, Fallgatter AJ. Functional near-infrared spectroscopy for the assessment of speech related tasks. Brain Lang. 2012;121(2):90-109. 
27. Ehlis AC, Schneider S, Dresler T, Fallgatter AJ. Application of functional near-infrared spectroscopy in psychiatry. Neuroimage. 2014;85(1): 478-488.

28. Aslin RN, Shukla M, Emberson LL. Hemodynamic correlates of cognition in human infants. Annu Rev Psychol. 2015;66:349-379.

29. Rizzolatti G, Sinigaglia C. The functional role of the parieto-frontal mirror circuit: interpretations and misinterpretations. Nat Rev Neurosci. 2010; 11(4):264-274.

30. Preston SD, de Waal FB. Empathy: its ultimate and proximate bases. Behav Brain Sci. 2002;25(1):1-20.

31. DavisMH. Measuring individual differences in empathy: evidence for a multidimensional approach. J Pers Soc Psychol. 1983;44(1):113-126.

32. Davis $\mathrm{MH}$. A multidimensional approach to individual differences in empathy. JSAS Catalog of Selected Documents in Psychology. 1980;10:85
33. Jabbi M, Swart M, Keysers C. Empathy for positive and negative emotions in the gustatory cortex Neuroimage. 2007;34(4):1744-1753.

34. Banissy MJ, Kanai R, Walsh V, Rees G. Inter-individual differences in empathy are reflected in human brain structure. Neuroimage. 2012;62(3): 2034-2039.

35. Ho SS, Konrath S, Brown S, Swain JE. Empathy and stress related neural responses in maternal decision making. Front Neurosci. 2014;8:152.

36. Kitano T. Takeshi Kitano Talks About a Scenario, Part 4- "BROTHER," “Dolls.” Narrative. Tokyo: rockin'on; 2012:103-137.

37. Keysers C, Gazzola V. Integrating simulation and theory of mind: from self to social cognition. Trends Cogn Sci. 2007;11(5):194-196.

38. Waytz A, Mitchell JP. Two mechanisms for simulating other minds: dissociations between mirroring and self-projection. Curr Dir Psychol Sci. 2011;20(3):197-200.

\section{Publish your work in this journal}

Neuroscience and Neuroeconomics is an international, peer-reviewed, open access journal focusing on the identification of brain structures and measurement of neural activity related to behavior, behavioral predictions, and decision making in health and disease. The manuscript management system is completely online and includes a very quick and fair peer-review system. Visit http://www.dovepress.com/testimonials. php to read real quotes from published authors. 